\title{
Apparatus for the Dynamic Calibration of Low-Range Differential Pressure Transducers
}

\author{
Mark A. Feero, Philippe Lavoie, and Pierre E. Sullivan \\ Version Post-print/accepted manuscript \\ Citation Feero, Mark A., Philippe Lavoie, and Pierre E. Sullivan. "Apparatus for \\ (published version) the Dynamic Calibration of Low-Range Differential Pressure \\ Transducers." AIAA Journal (2017): 1-4. Doi: 10.2514/1.J056373 \\ Publisher's Statement Copyright (C) 2017 by the American Institute of Aeronautics and \\ Astronautics, Inc. All rights reserved. All requests for copying \\ and permission to reprint should be submitted to CCC at \\ www.copyright.com; employ the ISSN $0001-1452$ (print) or 1533- \\ $385 X$ (online) to initiate your request. See also AIAA Rights and \\ Permissions www.aiaa.org/randp. \\ Read More: https://arc.aiaa.org/doi/abs/10.2514/1.J056373
}

How to cite TSpace items

Always cite the published version, so the author(s) will receive recognition through services that track citation counts, e.g. Scopus. If you need to cite the page number of the author manuscript from TSpace because you cannot access the published version, then cite the TSpace version in addition to the published version using the permanent URI (handle) found on the record page.

This article was made openly accessible by $U$ of $T$ Faculty. Please tell us how this access benefits you. Your story matters. 


\title{
Apparatus for the Dynamic Calibration of
}

\section{Low-Range Differential Pressure Transducers}

\author{
Mark A. Feero ${ }^{\mathrm{a}}$, Philippe Lavoie $^{\mathrm{b}}$ and Pierre E. Sullivan ${ }^{\mathrm{c}}$ \\ University of Toronto, Toronto, Ontario, Canada
}

\section{Introduction}

The development of an apparatus for the dynamic calibration of differential pressure transducers was motivated by the need to measure unsteady surface pressures on airfoils/wings during low speed wind tunnel experiments. In applications such as flow control or the study of dynamic stall, it is desirable to measure both the mean and fluctuating pressure at points on the airfoil surface. Accurate measurement of low frequency and low amplitude unsteady hydrodynamic pressure fluctuations is inherently difficult using the available measurement devices. The low amplitude and low frequency fluctuations inherent to the flow can be demonstrated by considering the freestream dynamic pressure, $q_{\infty}=0.5 \rho U_{\infty}^{2}$ (where $\rho$ is density and $U_{\infty}$ is freestream velocity), and characteristic frequency associated with flow over an airfoil. In the case of flow over a fixed airfoil, the lowest frequency of importance typically corresponds to the transit time over the airfoil surface, i.e., $f_{T}=U_{\infty} / c$, where $c$ is chord length. Figure 1 shows $q_{\infty}$ and $f_{T}$ for chord lengths and Reynolds numbers, $R e_{c}=U_{\infty} c / \nu$ (where $\nu$ is kinematic viscosity), typical of low Reynolds number wind tunnel studies at ambient temperature and pressure. Over this range of $R e_{c}$ and $c$, the freestream dynamic pressure is primarily below $1 \mathrm{kPa}$ and can be as low as $\sim 3 \mathrm{~Pa}$. Furthermore, RMS surface pressure fluctuations will typically have magnitudes at least an order of magnitude smaller than $q_{\infty}$ for incompressible flow at low Reynolds number (e.g., [1]). It can be observed that $f_{T}$ falls below the limit of "infrasound" (frequencies below $20 \mathrm{~Hz}$ ) for a wide range of experimental conditions. Studies involving unsteady airfoil motion often pitch the airfoil at frequencies considerably smaller

\footnotetext{
a Doctoral Candidate, Institute for Aerospace Studies, 4925 Dufferin Street, M3H 5T6.

b Associate Professor, Institute for Aerospace Studies, 4925 Dufferin Street, M3H 5T6.

c Professor, Department of Mechanical \& Industrial Engineering, 5 King's College Road, M5S 3G8.
} 
than $f_{T}$ (e.g., [2]), and therefore require the ability to measure relatively slow pressure fluctuations.

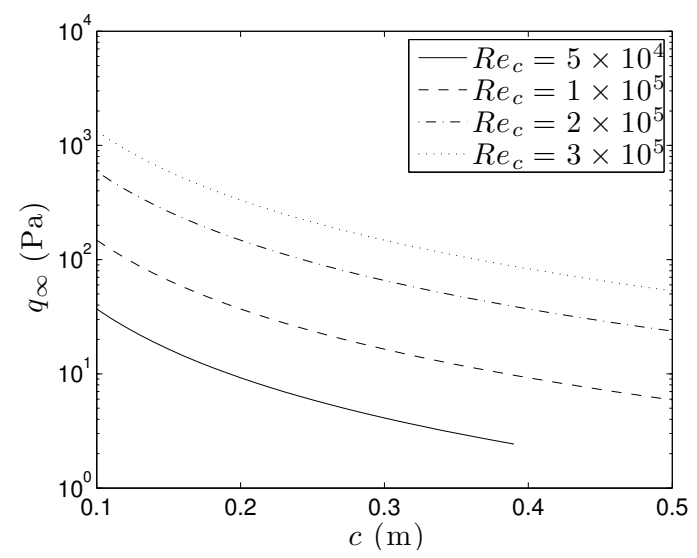

(a)

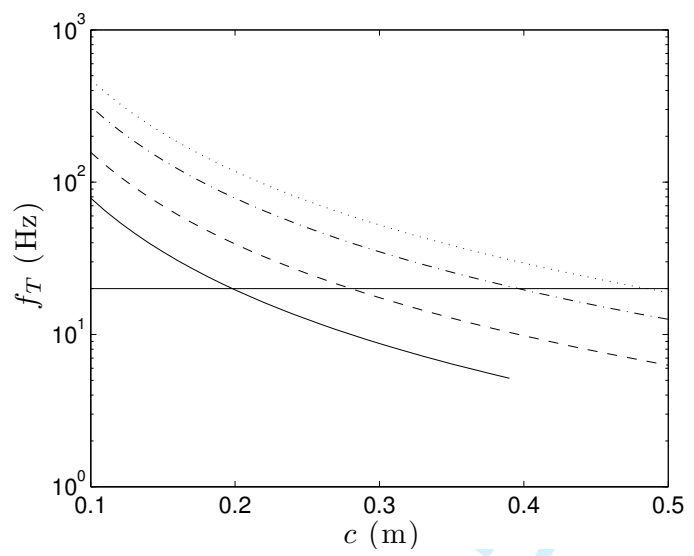

(b)

Fig. 1: Freestream dynamic pressure and transit-time frequency typical of low Reynolds number airfoil experiments in air at ambient temperature and pressure. The solid horizontal line in (b) denotes the upper limit of infrasound.

The difficulty arises when a measurement device must be selected to measure unsteady surface pressure under these conditions. Microphones are an obvious choice for measuring pressure fluctuations, however they do not measure the DC pressure and small electret microphones that could be implemented inside a model are not intended for measurements in the infrasound range. As such, their magnitude response decreases sharply at low frequency. Dynamic pressure transducers are ideal as they have a frequency response that is flat from DC up to a limit typically $\mathcal{O}(10) \mathrm{kHz}$, how- 
ever commercially available devices (e.g., Kulite) do not provide sufficient signal-to-noise for such low amplitude pressure fluctuations. Sensor size may also be a concern for installation within models of small size. Fuertes et al. [3] suggested a method that combines a microphone and a conventional differential pressure transducer (DPT) to measure the fluctuating and mean components of pressure, respectively. A pressure tap at the surface is connected to the DPT by a long length of tubing, and near the surface a microphone is connected to the tubing using a T-junction. This technique requires stationarity of the measured signal and dynamic calibration to account both for the microphones low frequency response and its position within the tubing. An alternative to this method is to only use a DPT for unsteady pressure measurement. These sensors have the advantage of being readily available with full-scale ranges suitable for low speed wind tunnel experiments. Such sensors are intended for the measurement of mean pressures, and as such generally have unknown dynamic characteristics. In addition to this, the geometry of the sensor itself and the tubing that is most often required for connection to the surface distorts pressure fluctuations, as described by Bergh and Tijdeman [4]. Dynamic calibration is therefore required to determine the frequency response of the DPT/tubing system. The experimentally measured transfer function or an analytically fit transfer function can then be used to correct measured pressure signals [5-7]. In some cases, it may also be possible to optimize the tubing geometry to produce a response that is flat from DC up to the largest frequency of interest [8] and no signal correction is required.

The most common types of equipment used for dynamic calibration of pressure transducers are aperiodic devices; shock tubes and fast opening pressure chambers. These comparative calibration devices expose the transducer and a reference sensor to a pressure step function. While these devices are suitable for a wide range of frequencies, they typically operate with large static pressures and large pressure step amplitudes (on the order of $\mathrm{kPa}$ to $\mathrm{MPa}$ ) [7, 9]. More appropriate for the calibration of low-range DPTs is a periodic device where either the volume or the mass inside a cavity is varied with time, thereby creating pressure fluctuations. Holmes and Lewis [8] used an electro-pneumatic valve to supply periodic, non-sinusoidal pressure to a cavity. Typically, pressure fluctuations are created inside a cavity volume using a speaker as one of the cavity walls [3, 10, 11]. Difficulties may be experienced with this calibration setup as common audio speakers often have 
a natural frequency below $100 \mathrm{~Hz}$, which is likely within the frequency range of interest (Figure 1), and below this the response is significantly attenuated. This work presents a compact, easily manufactured calibration apparatus that uses an oscillating piezoelectric disk rather than a speaker to create low amplitude pressure fluctuations inside a sealed cavity. Results are presented which demonstrate the calibration of a DPT using a microphone as reference.

\section{Experimental Setup}

The dynamic calibration apparatus is shown in Figure 2. Pressure fluctuations are created in a small cylindrical cavity with $30 \mathrm{~mm}$ diameter and $3.5 \mathrm{~mm}$ height by a piezoelectric disk that is clamped around its edge and forms one wall of the cavity. The cavity was designed to match the diameter of the piezoelectric disk, and the cavity height was kept small to ensure the displacement of the piezo was sufficient to create the desired pressure amplitudes. The TH-5C piezoelectic disk (Face International) has a diameter of $32.6 \mathrm{~mm}$ and a natural frequency of approximately $2500 \mathrm{~Hz}$. Compared with conventional audio speakers, which have poor response at low frequency, piezoelectric disks can operate from DC up to the kilohertz range. Input signals to the piezo disk are created by a function generator and are amplified by a Mide QPA3202 50:1 power amplifier. Pressure inside the cavity is measured both by the DPT to be calibrated and a GRAS 40BP $1 / 4$ " externally polarized pressure microphone with rear venting. This microphone, which served as the reference measurement, has a sensitivity of $1.6 \mathrm{mV} / \mathrm{Pa}$ and a flat response within $\pm 1 \mathrm{~dB}$ over $10 \mathrm{~Hz}-25 \mathrm{kHz}$. The microphone was connected to the cavity with its diaphragm flush with the cavity wall (the protective grid was removed) using a sealed fitting. The rear vent port was exposed to atmosphere. The example calibration was of an All Sensors 1 INCH-D-4V differential pressure transducer with $\pm 249 \mathrm{~Pa}$ range and accuracy of $\pm 0.12 \mathrm{~Pa}$. This DPT was selected to due its small size (approximately $12 \times 20 \times 30 \mathrm{~mm}^{3}$ ) and low relative cost, making it suitable for an application where a number of sensors could be installed within a wind tunnel model. The diameter and length of the tubing used to connected the DPT to the cavity was interchangeable. Connection of the tubing to the cavity was with a $0.5 \mathrm{~mm}$ diameter and $2 \mathrm{~mm}$ long pressure tap, which is representative of what may be used on an airfoil model. The reference port of the DPT was connected to a $2 \mathrm{~m}$ long length of tubing that was exposed to atmosphere on the opposite end. The manufacturer supplied 
1

2

3

4

5

6

7

8

9

10

11

12

13

14

15

16

17

18

19

20

21

22

23

24

25

static calibration of the DPT was verified by measuring the mean dynamic pressure in a wind tunnel using a pitot-static tube over a range of freestream velocities. An MKS Baratron 226A pressure transducer with $\pm 0.3 \%$ of-reading accuracy served as reference for the static calibration.

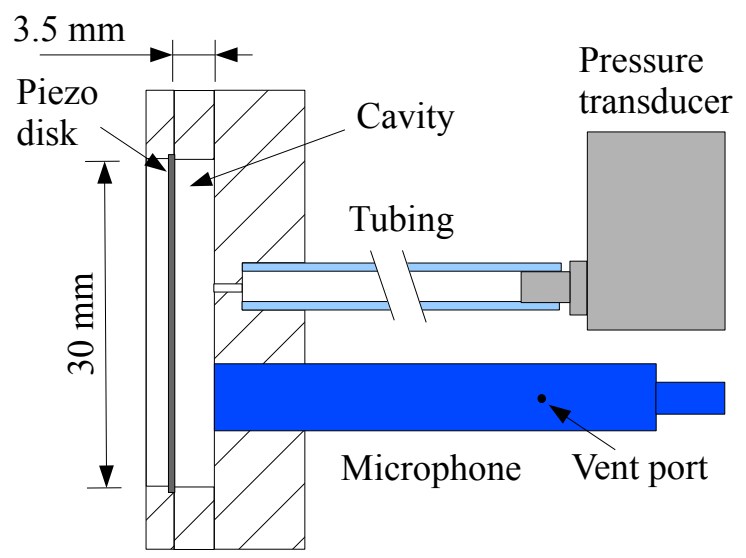

Fig. 2: Cross-sectional schematic of the dynamic calibration apparatus.

The input signal used to drive the piezo disk was a logarithmic sweep over $f=10-2000 \mathrm{~Hz}$. The lower frequency limit represents the limit of the reference microphone. The upper limit was set at $f=2000 \mathrm{~Hz}$, corresponding to an acoustic wavelength of $170 \mathrm{~mm}$, which is an order of magnitude larger than the largest linear dimension of the cavity. Therefore, it was assumed that the pressure at the tap and microphone diaphragm was equal over the swept frequency range. The input signal from the function generator, microphone output, and DPT output were sampled simultaneously at $5 \mathrm{kHz}$. The length of the sample was selected such that $N_{r}=100$ independent non-overlapping records (equivalent to the number of sweeps) each containing $2^{13}$ data points were acquired, giving a spectral resolution of $0.6 \mathrm{~Hz}$. Spectra and cross-spectra of the signals were computed using a Hamming window and $50 \%$ overlap between records.

\section{Results}

The calibration apparatus was used to calibrate the All Sensors DPT connected to the cavity by a $25 \mathrm{~mm}$ long PVC tube with $1.6 \mathrm{~mm}$ inner diameter. A portion of the time series that shows one full sweep is provided in Figure 3, where $p_{1}$ and $p_{2}$ refer to the microphone and DPT pressure, respectively. Figure 4 shows the power spectral density, $G_{p p}$, of the microphone and DPT signals 
over the calibration range $10 \leq f \leq 2000 \mathrm{~Hz}$. Over the majority of this frequency range, the power spectrum of the microphone is relatively flat and indicates that the fluctuating pressure amplitude does not vary considerably. As expected for a tubing/pressure transducer system, the DPT power spectrum shows a resonant peak, in this case occurring at $675 \mathrm{~Hz}$. A sharp decrease in the microphone power spectrum is observed below approximately $20 \mathrm{~Hz}$ where it falls below that of the DPT. This suggests that either these low frequency fluctuations are being correctly measured by the microphone and amplified by the DPT, or measured correctly by the DPT and attenuated by the microphone. An amplification at such low frequencies in addition to the resonant peak at 675 $\mathrm{Hz}$ is not expected based on models of the tubing/transducer system [4]. While frequencies down to $10 \mathrm{~Hz}$ are within the $\pm 1 \mathrm{~dB}$ range of the microphones frequency response, the issue is likely due to pressure fluctuations transmitted to the rear of the microphone diaphragm through the vent port. The air resistance within the small vent port is sufficient to prevent acoustic waves from entering the microphone cavity for most frequencies, however it is likely that low frequency waves emanating from the vibrating piezo disk are acting on the rear of the diaphragm. This causes the microphone to measure an apparent decrease in pressure amplitude. As such, the frequency response shown in Figure 5 has been corrected to be flat in this range. A unity gain corresponds to the sensitivity being equivalent to the $\mathrm{DC}$ sensitivity obtained from the static calibration $(0.8 \mathrm{mV} / \mathrm{Pa}$ in this case). The calibration apparatus produces a smooth frequency response that shows the expected behaviour for this system; a resonant peak associated with the tubing/transducer geometry followed by the attenuation of fluctuations at larger frequencies.

The quality of the calibration can be assessed by several metrics: the signal-to-noise ratio, $S / N$, of each signal, and the coherence between the two signals. The coherence is defined as

$$
\gamma_{p_{1} p_{2}}=\frac{\left|G_{p_{1} p_{2}}\right|^{2}}{G_{p_{1} p_{1}} G_{p_{2} p_{2}}}
$$

where $G_{p_{1} p_{2}}$ is the cross-spectral density of the signals. For the signal-to-noise ratio, noise signals were measured in a quiet environment with no excitation of the piezoelectric disk. Figure 6 shows $S / N$ of each signal and $\gamma_{p_{1} p_{2}}$ for the same calibration described above. The amplitude of pressure fluctuations in the cavity was approximately $65 \mathrm{~Pa}$ over the calibration frequency range. The results 

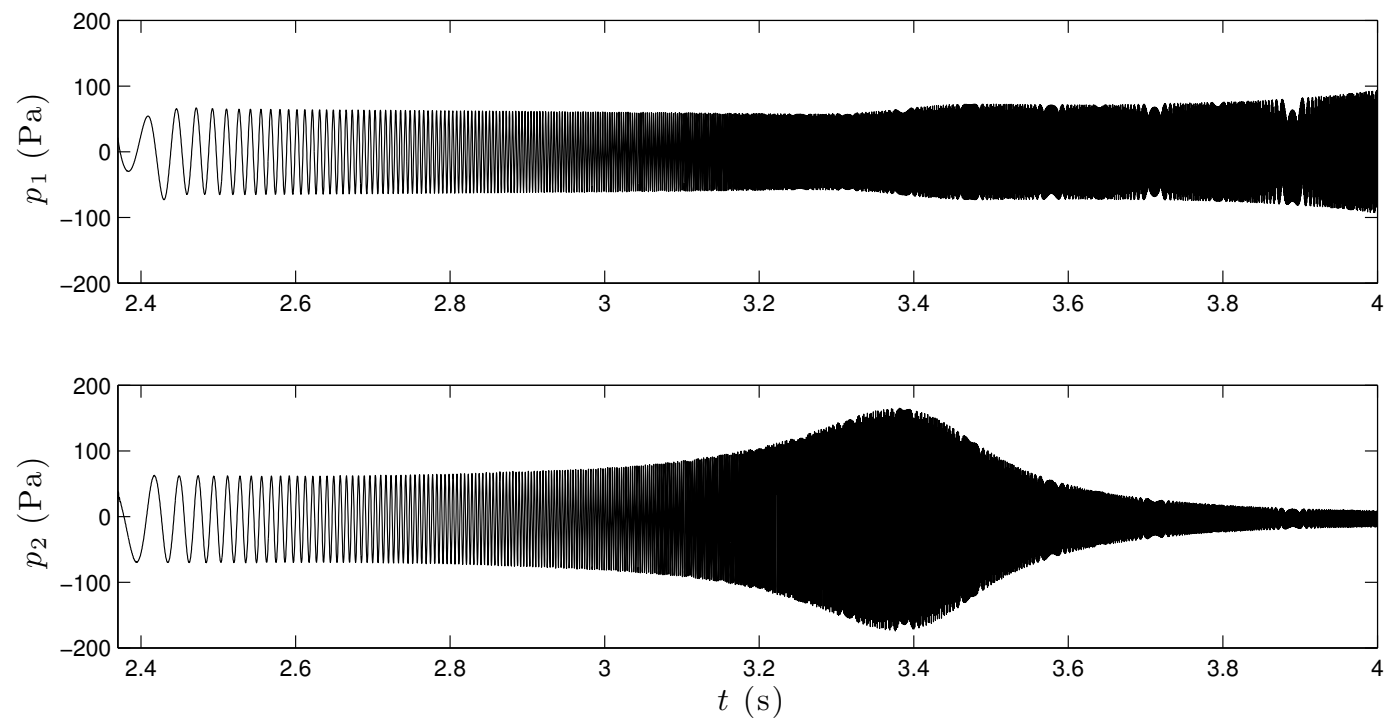

Fig. 3: Time series of the microphone and DPT pressure signals.

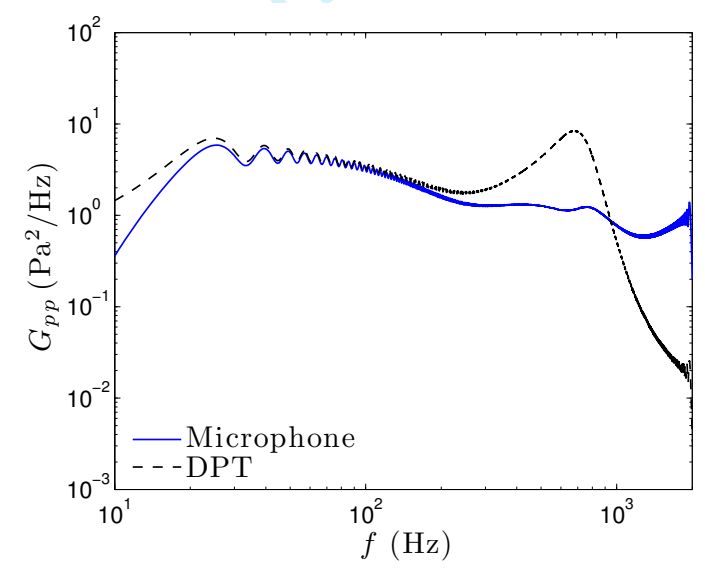

Fig. 4: Power spectra of the microphone and DPT signals.

demonstrate that $S / N$ for the microphone and DPT are at least $40 \mathrm{~dB}$ and $30 \mathrm{~dB}$, respectively, and thus each sensor is measuring well above its noise floor. The coherence demonstrates the linear relationship between the pressure measured by the microphone and the DPT, where unity coherence indicates a perfect linear relationship. The coherence is an important indicator of calibration quality since the random error in the frequency response is a function of $\gamma_{p_{1} p_{2}}[12]$, viz. 

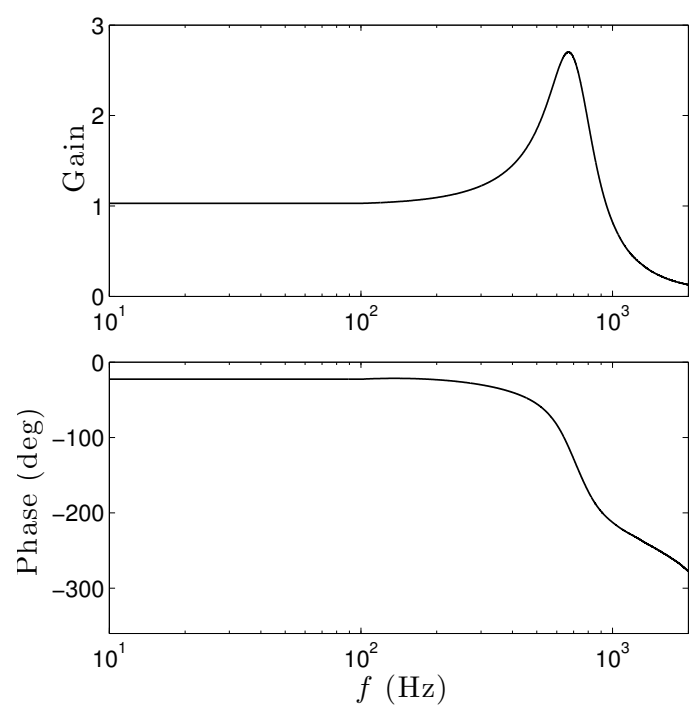

Fig. 5: Frequency response of the tubing/DPT system.

$$
\epsilon=\sqrt{\frac{1-\gamma_{p_{1} p_{2}}}{2 \gamma_{p_{1} p_{2}} N_{r}}},
$$

where $\epsilon$ is the relative error in gain and also the absolute standard deviation in phase given in radians. Therefore, the random error in the frequency response will be small even with few records for ensemble averaging for a calibration with $\gamma_{p_{1} p_{2}}(f) \approx 1$. Equation 2 is defined for spectra computed using rectangular, non-overlapping windows and serves only as an approximation of the random error for the present methodology (i.e., Hamming windows with $50 \%$ overlap). As shown in Figure 6 , the coherence is 1 over most of the frequency range with a minimum value of $\gamma_{p_{1} p_{2}}=0.987$. From equation (2), the random errors in gain and phase are less than $1 \%$ and $1^{\circ}$, respectively, over $10 \leq f \leq 2000 \mathrm{~Hz}$. It is of particular importance that despite the issue associated with the reference microphone at frequencies below $\sim 20 \mathrm{~Hz}$, accurate calibration at low frequencies relevant to Figure $1 \mathrm{~b}$ is possible with this apparatus. Calibration with low error is achieved even in the frequency range above $1000 \mathrm{~Hz}$ where the pressure signal measured by the DPT is strongly attenuated. It is important to note that this is not only due to the high spectral coherence due to the calibration apparatus, but is also a result of a sufficiently high signal-to-noise ratio for both the DPT being calibrated and the reference microphone. 
1

2

3

4

5

6

7

8

9

10

11

12

13

14

15

16

17

18

19

20

21

22

23

24

25

26

27

28

29

30

31

32

33

34

35

36

37

38

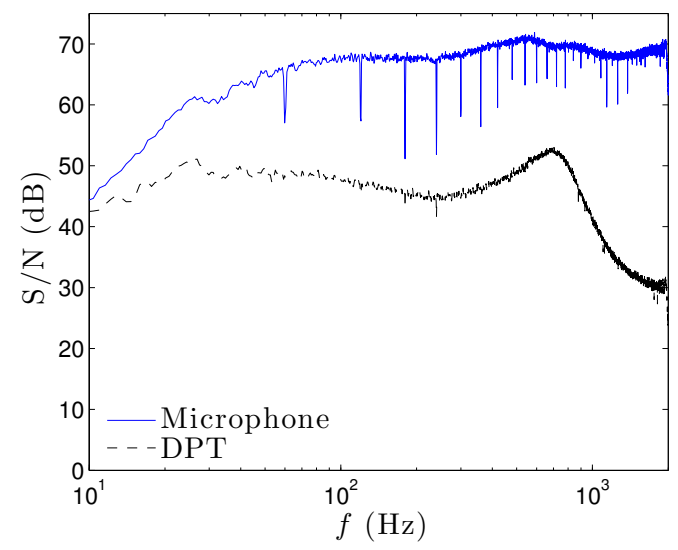

(a)

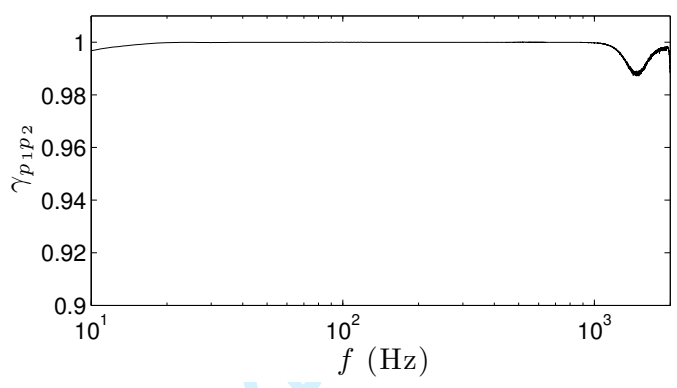

(b)

Fig. 6: Signal-to-noise and spectral coherence for the same calibration as Figure 5.

\section{Conclusions}

A simple device for the dynamic calibration of low-range differential pressure transducers has been presented. This calibration apparatus employs periodic pressure fluctuations created inside a cavity by an oscillating piezoelectic disk. The apparatus is easily manufactured and is well suited for calibrating low-range pressure sensors for use in applications such as low Reynolds number airfoil experiments in subsonic wind tunnels. An example calibration was presented for a DPT with a range of $\pm 249 \mathrm{~Pa}$ and a pressure microphone with a sensitivity of $1.6 \mathrm{mV} / \mathrm{Pa}$ served as reference. At frequencies below approximately $20 \mathrm{~Hz}$, an artificial decrease in the magnitude of fluctuations measured by the microphone was observed, which was attributed to low frequency acoustic waves affecting the microphone diaphragm through its rear ventilation port. Despite the low amplitude of the pressure fluctuations inside the cavity $(\sim 65 \mathrm{~Pa})$, the calibration apparatus was found to 
produce a smooth frequency response for the DPT. The estimated random errors in the frequency response magnitude and phase were found to be less than $1 \%$ and $1^{\circ}$, respectively, due to high coherence between signals.

\section{References}

[1] Boutilier, M. S. and Yarusevych, S., "Separated shear layer transition over an airfoil at a low Reynolds number," Physics of Fluids, Vol. 24, No. 8, 2012, pp. 084105.

[2] Rival, D. and Tropea, C., "Characteristics of pitching and plunging airfoils under dynamic-stall conditions," Journal of Aircraft, Vol. 47, No. 1, 2010, pp. 80-86.

[3] Fuertes, F. C., Cecchi, E., van Beeck, J., and Schram, C., "An inexpensive and versatile technique for wide frequency range surface pressure measurements: an application for the study of turbulent buffeting of a square cylinder," Experiments in fluids, Vol. 55, No. 1, 2014, pp. 1627.

[4] Bergh, H. and Tijdeman, H., "Theoretical and experimental results for the dynamic response of pressure measuring systems," Tech. rep., Nationaal Lucht-en Ruimtevaartlaboratorium, 1965.

[5] Irwin, H., Cooper, K., and Girard, R., "Correction of distortion effects caused by tubing systems in measurements of fluctuating pressures," Journal of Wind Engineering and Industrial Aerodynamics, Vol. 5, No. 1-2, 1979, pp. 93-107.

[6] Semaan, R. and Scholz, P., "Pressure correction schemes and the use of the Wiener deconvolution method in pneumatic systems with short tubes," Experiments in fluids, Vol. 53, No. 3, 2012, pp. 829837.

[7] Whitmore, S. A. and Wilson, M. D., "Wiener deconvolution for reconstruction of pneumatically attenuated pressure signals," AIAA journal, Vol. 49, No. 5, 2011, pp. 890-897.

[8] Holmes, J. and Lewis, R., "Optimization of dynamic-pressure-measurement systems. I. Single point measurements," Journal of Wind Engineering and Industrial Aerodynamics, Vol. 25, No. 3, 1987, pp. 249273.

[9] Damion, J., "Means of dynamic calibration for pressure transducers," Metrologia, Vol. 30, No. 6, 1994, pp. 743 .

[10] Bernardini, C., Benton, S. I., Hipp, K. D., and Bons, J. P., "Large Low-Frequency Oscillations Initiated by Flow Control on a Poststall Airfoil," AIAA Journal, Vol. 54, No. 1, 2016, pp. 1616-1627.

[11] Zakrzewski, J. and Wrobel, K., "Dynamic calibration of low-range silicon pressure sensors," IEEE Transactions on instrumentation and measurement, Vol. 51, No. 6, 2002, pp. 1358-1362. 
[12] Bendat, J. S. and Piersol, A. G., Random data: analysis and measurement procedures, Vol. 729, John Wiley \& Sons, 2011.

1

2

3

4

5

6

7

8

9

10

11

12

13

14

15

16

17

18

19

20

21

22

23

24

25

26

27

28

29

30

31

32

33

34

35

36

37

38

39

40

41

42

43

44

45

46

47

48

49

50

51

52

53

54

55

56

57

58

59

60 Submitted to AIAA Journal. Confidential - Do not distribute. 